\title{
Neural systems of visual attention responding to emotional gestures
}

\author{
Tobias Flaisch ${ }^{\mathrm{a}, *}$, Harald T. Schupp ${ }^{\text {a }}$, Britta Renner ${ }^{\text {a }}$, Markus Junghöfer ${ }^{\mathrm{b}}$ \\ a Department of Psychology, University of Konstanz, P.O. Box D 36, 78457 Konstanz, Germany \\ b Institute for Biomagnetism and Biosignalanalysis, Münster University Hospital, Malmedyweg 1, 48149 Münster, Germany
}

\begin{abstract}
A B S T R A C T
Humans are the only species known to use symbolic gestures for communication. This affords a unique medium for nonverbal emotional communication with a distinct theoretical status compared to facial expressions and other biologically evolved nonverbal emotion signals. While a frown is a frown all around the world, the relation of emotional gestures to their referents is arbitrary and varies from culture to culture. The present studies examined whether such culturally based emotion displays guide visual attention processes. In two experiments, participants passively viewed symbolic hand gestures with positive, negative and neutral emotional meaning. In Experiment 1, functional magnetic resonance imaging (fMRI) measurements showed that gestures of insult and approval enhance activity in selected bilateral visual associative brain regions devoted to object perception. In Experiment 2, dense sensor event related brain potential recordings (ERP) revealed that emotional hand gestures are differentially processed already $150 \mathrm{~ms}$ poststimulus. Thus, the present studies provide converging neuroscientific evidence that emotional gestures provoke the cardinal signatures of selective visual attention regarding brain structures and temporal dynamics previously shown for emotional face and body expressions. It is concluded that emotionally charged gestures are efficient in shaping selective attention processes already at the level of stimulus perception.
\end{abstract}

\section{Introduction}

In natural environments, emotional cues guide visual attention and receive priority processing (Lang et al., 1997; Öhman et al., 2000; Vuilleumier, 2005). The emotional attention capture seems highly adaptive facilitating the organization of efficient behaviors. Previous research determined emotional attention effects by studying stimulus materials which relate to agendas set by evolution, i.e., seeking protection and support from conspecifics, getting access to sexual partners, and avoiding life threatening dangers (Lang et al., 1997; Öhman et al., 2000). Here, we extend this line of research to the study of symbolic gestures with emotional meaning.

In face to face interactions, humans frequently use symbolic gestures to express emotional meaning. Within a split second, a specific gesture may show approval, calm down a heated exchange, or can signal strong insult, offense and threat (Morris, 1994). Accordingly, the study of emotional gestures provides new insights into nonverbal emotional communication. Compared to biologically evolved signals, gestures possess a distinct theoretical status by representing a socially shared signal system (Buck and VanLear, 2002). Gestures differ with respect to the relation of the sign to the signified meaning. Specifically, the relation of emotional gestures to

\footnotetext{
* Corresponding author. Fax: +49 7531882971.

E-mail address: tobias.flaisch@uni-konstanz.de (T. Flaisch).
}

their referents is arbitrary, builds upon shared meaning and convention, and consequently varies from culture to culture (Archer, 1997). Symbolic gestures are closely related to language and unique to humans, as chimpanzees' use of gestures lacks symbolic character and originates in dyadic ritualization (Pika et al., 2005). Accordingly, it needs to be determined whether emotional gestures capture visual attention similar to biologically shared emotion signals.

Previous neuroimaging studies revealed the attention capture of biologically shared emotion cues. The processing of pictures of erotica, mutilation and threat is associated with increased activations in visual cortical regions (i.e., occipital, parietal, and inferior temporal cortices; Bradley et al., 2003; Junghöfer et al., 2005, 2006; Sabatinelli et al., 2005). Furthermore, fearful, angry, and happy facial expressions reliably enhance the activation of the fusiform face area compared to neutral expressions (Pessoa et al., 2002; Vuilleumier et al., 2001). Similarly, fearful and threatening body expressions have been shown to enhance activation in fusiform gyrus and lateral occipito temporal cortex compared to neutral postures (de Gelder et al., 2004; Hadjikhani and de Gelder, 2003; Peelen et al., 2007). Taken together, it is suggested that emotional stimuli increase the activation of brain regions devoted to object perception.

Event related brain potentials allow to delineate the temporal dynamics of emotion processing in visual associative cortical regions. The processing of pictures of erotica, mutilation and threat is associated with an early posterior negativity (EPN), appearing around 
120150 ms poststimulus (Junghöfer et al., 2001; Schupp et al., 2003). Similar observations have been reported regarding the processing of threatening and fearful compared to neutral facial expressions (Leppänen et al., 2007; Sato et al., 2001; Schupp et al., 2004). Interestingly, explicit attention to distinct stimulus features or higher order categorizations defined by semantic meaning is associated with a similar ERP difference (Codispoti et al., 2006; Delorme et al., 2004; Potts and Tucker, 2001). Overall, emotional stimulus processing is facilitated during an early processing stage presumed to reflect selection for elaborate processing.

Utilizing functional magnetic resonance imaging (fMRI, Study 1) and event related brain potentials (ERP, Study 2), two studies investigated whether emotional hand gestures elicit the neural signature of visual attention. In both studies, participants passively viewed a rapid stream of three semantically meaningful hand gestures bearing positive, neutral and negative meaning in the German culture. Regarding neural structure, Study 1 explored the hypothesis that emotional compared to neutral gestures elicit enhanced brain activity in associative visual cortical regions. Regarding the speed of emotion processing, Study 2 examined the notion that the processing of emotional cues is associated with increased EPN amplitudes emerging around $120150 \mathrm{~ms}$ poststimulus. Further analyses provide comple mentary information regarding the attention capture of emotional gestures. Gender effects were explored to determine whether women and men respond differentially to unpleasant and pleasant gestures (Bradley et al., 2001; Sabatinelli et al., 2004). Additionally, habituation effects were assessed to determine whether the preferential proces sing of gestures is maintained across stimulus repetitions (Schupp et al., 2006).

\section{Materials and methods}

\section{Participants}

Thirty (16 females) and sixteen ( 8 females) volunteers aged between 19 and 33 years participated in Studies 1 and 2, respectively. The experimental procedures were approved by the ethical committee of the University of Konstanz and all participants provided informed consent. They received monetary compensation or course credit for participation.

\section{Stimuli}

A small set of gestures was selected to achieve a high level of control regarding several criteria: (1) distinct semantic meaning solely carried by a static hand gesture, (2) culturally shared meaning, (3) explicit emotional significance, and (4) perceptual similarity. An up raised middle finger, which is amongst the strongest hand signs of sexual insult, served as the negative gesture ('Insult'). An up raised thumb above a fist, which signals approval and is also referred to as the Ok sign, served as the positive gesture ('Ok'). The forefinger pointing horizontally from a fist served as the emotionally neutral control gesture ('Point'; Morris, 1994; Morris et al., 1979). Each gesture was posed by four women and four men. All gestures were displayed with the back of the hand rotated towards the viewer and with a neutral single colored gray blue background.

\section{Self report}

Following MR scanning and ERP measurement, participants were asked to rate the viewed gestures according to their perceived pleasantness and arousal using the Self Assessment Manikin (Bradley and Lang, 1994). For statistical analysis, both measures were entered into a one factorial repeated measure ANOVA with the factor Gesture (Insult vs. Ok vs. Point).

\section{Study 1: procedure, fMRI data acquisition and analysis}

Participants were instructed to passively view the experimental stimulation while being scanned. The three gestures and an interleaved fixation baseline condition were presented in blocks of $12 \mathrm{~s}$ each, which followed each other in a pseudo randomized order assuring adequate control of block sequence. Each gesture block contained the presentation of 16 stimuli, each shown for $330 \mathrm{~ms}$ immediately followed by a $420 \mathrm{~ms}$ period of a blank screen.

Over the entire experimental session, each category (Insult, Ok, Point, Fixation) was shown 14 times ( 7 times flipped horizontally to control for lateralization effects) resulting in 56 blocks overall.

Scanning was conducted using a $1.5 \mathrm{~T}$ Philips Intera MR System equipped with Power Gradients. For functional scanning, a T2* weighted Fast Field Echo, Echo Planar Imaging (FFE EPI) sequence utilizing parallel scanning technique was used (SENSE; Pruessmann et al., 1999). In plane resolution of the axially acquired slices was $2.9 \times 2.9 \mathrm{~mm}$ and the slice thickness was $3.5 \mathrm{~mm}$ (36 slices; no gap; FOV $=230 \mathrm{~mm}$; acquisition matrix: $80 \times 80$ voxels; $\mathrm{TE}=40 \mathrm{~ms}$; flip angle $=90^{\circ} ; \mathrm{TR}=3 \mathrm{~s}$ ). In addition, a standard $\mathrm{T} 1$ weighted high resolution structural scan was obtained for each participant.

Preprocessing (including slice time correction, realignment and spatial normalization) and statistical analyses of the functional images were conducted using Statistical Parametric Mapping (Friston et al., 1995; SPM5; Wellcome Department of Imaging Neuroscience, University College London, UK; http://www.fil.ion.ucl.ac.uk/spm/soft ware/spm5). No participant displayed head movement exceeding $1 \mathrm{~mm}$ on any axis and thus all were included in further analysis. The design matrix was comprised by three covariates of interest repre senting the experimental conditions' onsets and the duration of the different picture presentation epochs, as well as covariates of no interest including the modeled response functions' time and disper sion derivatives, six movement parameters obtained during realign ment and one covariate incorporating an overall intercept to the model. The fixation baseline was modeled implicitly. Before estimat ing the modeled regressors, a high pass filter with a cutoff period of $128 \mathrm{~s}$, as well as global scaling was applied to the data.

Following model estimation, planned contrasts of interest were calculated for each participant. A first stream of exploratory random effects analyses assessed expected activation increases by contrasting the emotional gestures with the neutral control category (Insult $>$ Point, Ok $>$ Point). To specifically test the hypothesis that emotional gestures are preferentially processed in higher order sensory brain regions, a subsequent region of interest (ROI) analysis focused on visual cortical areas consistently activated by hand gestures. For this purpose, comparisons of all experimental conditions with the fixation baseline were calculated (Insult $>$ Fixation, Ok $>$ Fixa tion, Point $>$ Fixation) and entered into a 2nd level random effects ANOVA. To derive focal functional ROIs representing visual activation common to all picture categories, a conjunction contrast applying stringent statistical criteria was calculated (statistical voxel threshold: $T>12$ ). This resulted in two lateralized anatomical cluster sites at occipito temporal and posterior mid occipital locations, respectively (see Table 1 and Fig. 2). The average of the model estimation parameters (betas) of all activated voxels within these ROIs was extracted for each participant and submitted to repeated measure ANOVAs including the factors Gesture (Insult vs. Ok vs. Point) and Laterality (left vs. right). In all reported analyses, the Greenhouse Geisser procedure was used to correct for violations of sphericity. Figures 2 and 3 were created using MRIcro software (Rorden and Brett, 2000; http://www.sph.sc.edu/comg/rorden/mricro.html).

\section{Experiment 2: procedure, ERP data acquisition and analysis}

The same stimuli were used as in Study 1. As in previous ERP research (Flaisch et al., 2008), the gestures were presented as a 
Table 1

Regional brain responses to emotional vs. neutral gestures in Study 1

\begin{tabular}{|c|c|c|c|c|c|c|c|}
\hline Region & Hemi & BA & $\mathrm{k}$ & $x$ & $y$ & $z$ & $\mathrm{~T}$ \\
\hline \multicolumn{8}{|l|}{ Insult $>$ Point } \\
\hline \multicolumn{8}{|l|}{ Parietal } \\
\hline Superior parietal lobule & $\mathrm{R}$ & 7 & 35 & 27 & -53 & 55 & 2.59 \\
\hline Inferior parietal lobule & $\mathrm{R}$ & 40 & 121 & 62 & -22 & 26 & 4.08 \\
\hline Precentral gyrus & $\mathrm{R}$ & 46 & 48 & 62 & -10 & 34 & 3.60 \\
\hline Postcentral gyrus & $\mathrm{R}$ & 1235740 & 362 & 39 & -44 & 57 & 3.68 \\
\hline \multicolumn{8}{|l|}{ Occipital } \\
\hline Precuneus & $\mathrm{R}$ & 7193139 & 92 & 30 & -65 & 31 & 4.36 \\
\hline \multirow[t]{2}{*}{ Cuneus } & $\mathrm{L}$ & 171819 & 208 & -9 & -95 & 24 & 4.57 \\
\hline & $\mathrm{R}$ & 7171819 & 244 & 21 & -95 & 24 & 6.30 \\
\hline Superior occipital gyrus & $\mathrm{R}$ & 1939 & 23 & 30 & -71 & 28 & 3.56 \\
\hline \multirow[t]{2}{*}{ Middle occipital gyrus } & $\mathrm{L}$ & 181937 & 355 & -45 & -76 & -4 & 6.02 \\
\hline & $\mathrm{R}$ & 181937 & 480 & 48 & -67 & -7 & 7.03 \\
\hline \multirow[t]{2}{*}{ Inferior occipital gyrus } & $\mathrm{L}$ & 1819 & 67 & -45 & -79 & -6 & 5.33 \\
\hline & $\mathrm{R}$ & 171819 & 122 & 45 & -76 & -6 & 6.63 \\
\hline \multicolumn{8}{|l|}{ Temporal } \\
\hline \multirow[t]{2}{*}{ Middle temporal gyrus } & $\mathrm{L}$ & 193739 & 34 & -48 & -78 & 12 & 3.23 \\
\hline & $\mathrm{R}$ & 1921223739 & 159 & 53 & -59 & -7 & 4.65 \\
\hline \multirow[t]{2}{*}{ Inferior temporal gyrus } & $\mathrm{L}$ & 181937 & 43 & -48 & -76 & -1 & 5.82 \\
\hline & $\mathrm{R}$ & 181937 & 79 & 48 & -73 & -1 & 8.44 \\
\hline \multirow[t]{2}{*}{ Fusiform gyrus } & $\mathrm{L}$ & 193637 & 77 & -48 & -68 & -12 & 4.65 \\
\hline & $\mathrm{R}$ & 1937 & 171 & 48 & -65 & -12 & 5.13 \\
\hline \multicolumn{8}{|l|}{ Ok $>$ Point } \\
\hline \multicolumn{8}{|l|}{ Occipital } \\
\hline \multirow[t]{2}{*}{ Cuneus } & $\mathrm{L}$ & 1819 & 152 & -9 & -95 & 25 & 4.67 \\
\hline & $\mathrm{R}$ & 1819 & 149 & 6 & -89 & 21 & 4.28 \\
\hline \multirow[t]{2}{*}{ Middle occipital gyrus } & $\mathrm{L}$ & 18 & 24 & -9 & -101 & 16 & 5.21 \\
\hline & $\mathrm{R}$ & 1819 & 25 & 9 & -95 & 13 & 4.61 \\
\hline
\end{tabular}

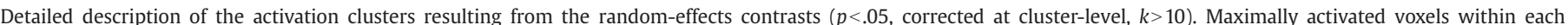

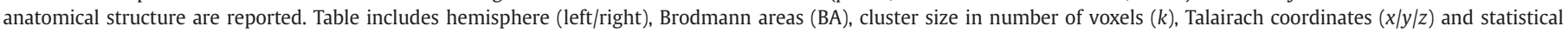
values $(T)$. Anatomical localization was determined using the Talairach daemon software (Lancaster et al., 2000; http://www.talairach.org/).

continuous stream without perceivable interstimulus interval for $330 \mathrm{~ms}$ each. The entire picture set was repeated 45 times resulting in a total of 1080 picture presentations. The pictures were shown in a randomized order in which no more than three pictures of the same gesture category were presented in succession and the transition frequencies between all categories were controlled.

Brain and ocular scalp potential fields were measured with a 256 lead geodesic sensor net (GSN 200 v2.0; EGI: Electrical Geodesics, Inc., Eugene, OR), on line bandpass filtered from $0.01100 \mathrm{~Hz}$, and sampled at $250 \mathrm{~Hz}$ using Netstation acquisition software and EGI amplifiers. Data editing and artifact rejection were based on an elaborate method for statistical control of artifacts, specifically tailored for the analysis of dense sensor ERP recordings (Junghöfer et al., 2000; Junghöfer and Peyk, 2004). The reported data were baseline adjusted and converted to an average reference. Finally, separate average waveforms were calculated for the three gesture categories, for each sensor and participant.

In a first exploratory step, single sensor waveform analyses were used to determine time course and topographical distribution of modulatory effects of the different gestures (Schupp et al., 2007). Based on this, mean amplitudes from occipito temporal sensor clusters were calculated in the time window from $200300 \mathrm{~ms}$. Subsequently, these were submitted to a conventional two factorial repeated measure ANOVA with the factors Gesture (Insult vs. Ok vs. Point) and Laterality (left vs. right). When appropriate, the Green house Geisser procedure was used to correct for violations of sphericity.

Calculation of the L2 Minimum Norm was based on a four shell spherical head model with evenly distributed 3 (radial, azimuthal and polar direction) $\times 350$ dipoles as source model (Hauk, 2004). A source shell radius of $87 \%$ of the spherical volume conductor head radius has been chosen, roughly corresponding to the grey matter volume. Across all participants and conditions, a Tikhonov regularization parameter $k$ of 0.02 was applied.

\section{Results}

Study 1: fMRI

\section{Self report ratings}

As shown in Fig. 1A, highly significant main effects for Gesture were observed for valence $(F(2,58)=228.9, p<.001, \varepsilon=0.95)$ and arousal ratings $(F(2,58)=16.7, p<.001, \varepsilon=0.98)$. With regard to valence, the Insult gesture was evaluated negatively and the Ok gesture positively (Insult vs. Ok: $t(29)=19.5, p<.001$; Insult vs. Point: $t(29)=8.1, p<.001$; Ok vs. Point: $t(29)=14.6, p<.001)$. With regard to arousal, the Insult gesture was rated as more arousing than the Ok $(t(29)=4.6, p<.001)$ and Point gesture $(t(29)=5.4, p<.001)$, which received similar arousal ratings.

\section{Random effects analysis}

A first set of analyses explored expected activation differences by contrasting emotional and neutral gestures. The Insult gesture elicited robust activations in temporo and parieto occipital brain regions compared to the Point gesture, and this effect was stronger in the right hemisphere. Pronounced increases in BOLD activity were observed in bilateral secondary visual cortical and right hemisphere parietal areas (see Table $1 ; p<.05$, corrected at cluster level, minimum cluster size: $k>10$ ). In comparison, differences between the Ok and Point gesture were less pronounced (see Fig. 2 and Table 1). However, data exploration relying on a liberal statistical threshold $(p<.01$, uncor rected, $k>30$ ) revealed similar activation differences in visual cortical areas as were seen when comparing the Insult with the Point gesture (see Fig. 2).

\section{Region of interest analysis}

A more stringent test of the hypothesis that emotional gestures attract selective visual attention is to focus on brain regions consistently activated by hand gestures and test for differences 
A

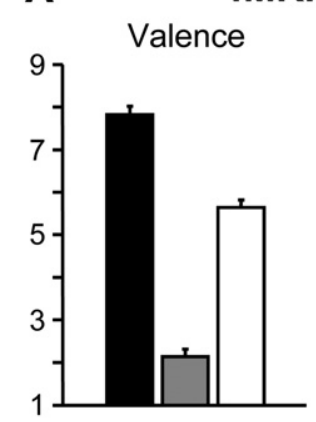

B

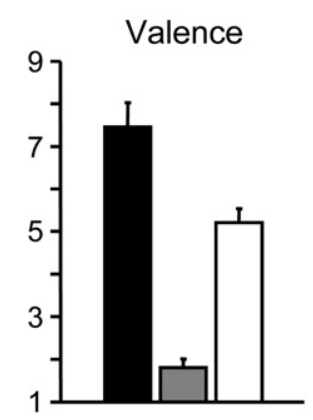

Arousal

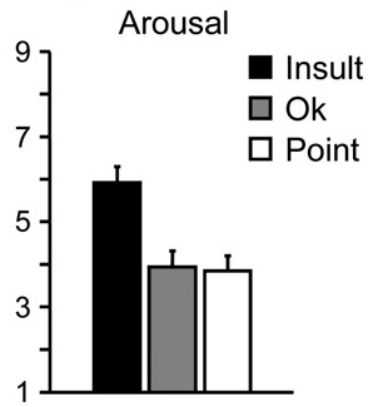

EEG-Study

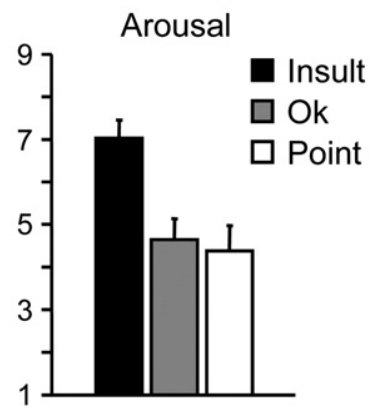

Fig. 1. Mean valence and arousal ratings as a function of gesture type for Studies 1 and 2. Hedonic valence increased linearly from Ok to Point and from the Point to the Insult gesture ( 1 =most pleasant; $9=$ most unpleasant). Emotional arousal was enhanced for the Insult gesture compared to the Point and the Ok gesture which did not differ ( 1 =least arousing; $9=$ most arousing). Error bars represent standard errors of the mean.

among the gestures within these regions of interest. A conjunction analysis revealed that hand gestures elicited robust activations in a posterior mid occipital region comprising activity in the cuneus and middle occipital gyrus, as well as in the lateral occipito temporal cortex corresponding to object selective areas in the brain (see Fig. 3).

\section{Lateral occipito temporal ROI}

Emotional modulation effects appeared more pronounced over the right hemisphere, Gesture $\times$ Laterality $(F(2,58)=10.3, p<.001, \varepsilon=0.97$; see Fig. 3A). The separate analysis of the left side revealed a significant main effect of Gesture $(F(2,58)=16.3, p<.001, \varepsilon=0.98)$. The Insult and Ok gesture elicited increased activity compared to the Point gesture (Insult vs. Point: $t(29)=6.2, p<.001$; Ok vs. Point: $t(29)=2.5, p<.05$ ), and the effect was most pronounced for the Insult gesture (Insult vs Ok: $t(29)=3.0, p<.01)$. Analysis of the right occipito temporal ROI revealed a highly significant main effect of Gesture $(F(2,58)=52.2$, $p<.001, \varepsilon=0.9$ ). Similar to the left ROI, the Insult gesture elicited increased activity relative to the Point $(t(29)=9.0, p<.001)$, and Ok gesture $(t(29)=7.2, p<.001)$, and the Ok gesture elicited moderately increased activation compared to the Point gesture $(t(29)=2.0, p=.05)$.

\section{Posterior mid occipital ROI}

A significant interaction between Gesture and Laterality $(F(2,58)=$ 6.9, $p<.01, \varepsilon=0.96$; see Fig. 3B) was obtained, followed up by analyzing left and right mid occipital ROIs separately. In the left hemisphere, activation was significantly modulated by Gesture ( $F(2$, $58)=8.7, p<.001, \varepsilon=0.94)$. Separate tests revealed that the Insult gesture elicited increased activity compared to both other gestures (Insult vs. Point: $t(29)=3.7, p<.001$; Insult vs. Ok: $t(29)=3.7, p<.001$ ), which did not differ from each other (Ok vs. Point: $t(29)=0.6$, ns). In the right hemisphere, both emotional gestures elicited enhanced activity compared to the Point gesture $(F(2,58)=48.4, p<.001$, $\varepsilon=0.99)$. Specifically, the Insult gesture yielded increased activation

relative to the $\mathrm{Ok}(t(29)=9.9, p<.001)$ and Point gesture $(t(29)=5.7$, $p<.001)$. Furthermore, processing of the Ok gesture also elicited increased activity in the mid occipital ROI compared to the Point gesture $(t(29)=4.0, p<.001)$.

\section{Gender effects}

The large sample allowed to additionally explore whether these effects differed for men and women. Incorporating the Gender factor in these analyses confirmed the preferential processing of emotional gestures in the occipito temporal and mid occipital ROIs for both genders and no significant interaction involving Gender was obtained.

\section{Habituation effects}

One hypothesis to explain more pronounced activation effects for the Insult than for the Ok gesture is to assume that responding to the insulting gesture is more sustained. To examine habituation effects, analyses were repeated with the additional factor Time (1st half vs. 2nd half). Similar effects for emotional gesture processing were observed for the first and second half of the experiment in both ROIs, and no higher order interaction involving Time reached significance.

Study 2: EEG

\section{Self report ratings}

Analyses fully replicated findings from the fMRI Study (see Fig. 1B). Specifically, highly significant Gesture main effects for valence $(F(2$, $30)=46.1, p<.001, \varepsilon=0.75)$ and arousal $(F(2,30)=10.7, p<.01, \varepsilon=0.77)$ were observed. With regard to valence, the Insult gesture was evaluated negatively and the Ok gesture positively (Insult vs. Ok: $t$ $(15)=8.1, p<.001$; Insult vs. Point: $t(15)=3.5, p<.01$; Ok vs. Point: $t$ $(15)=8.7, p<.001)$. With regard to arousal, the Insult gesture was rated as more arousing than the $\mathrm{Ok}(t(15)=5.2, p<.001)$ and Point gesture $(t$ $(15)=4.2, p<.001)$, which received similar arousal ratings.

\section{Event related potentials}

The Insult gesture was associated with a relative negative deflection compared to both the Ok and Point gestures (see Fig. 4B). This effect was most pronounced over the right hemisphere, developed around $150 \mathrm{~ms}$ and reached its maximum between 200 and $300 \mathrm{~ms}$. Although less pronounced, the Ok gesture also elicited an enlarged negativity compared to the Point gesture. Furthermore, as expected according to biophysical principles of electric field poten tials, polarity reversal was observed over anterior sensor locations, mirroring the effects over posterior sites.

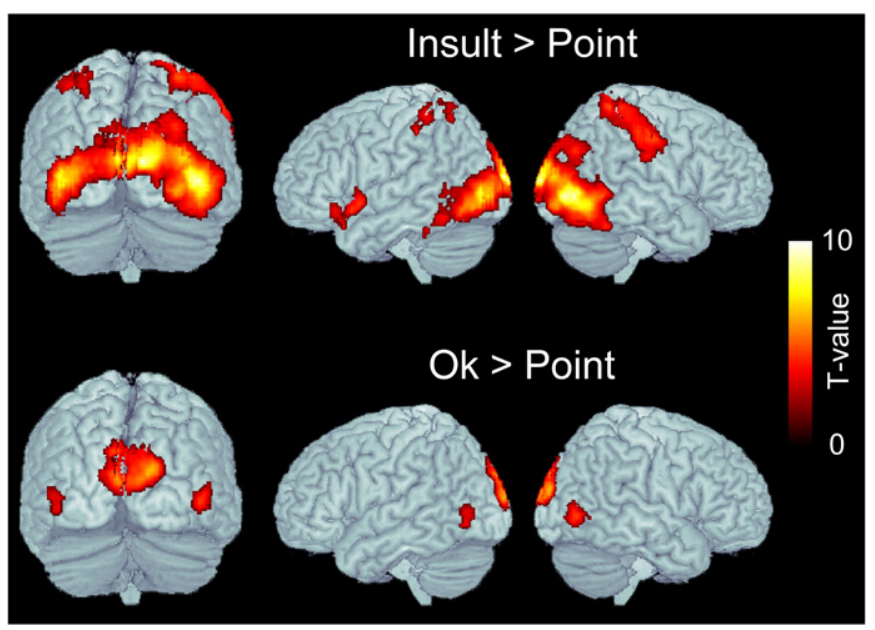

Fig. 2. Localization of increased BOLD-activation contrasting the Insult and Ok gestures against the emotionally neutral Point gesture, respectively. For illustrative purposes, activations are displayed at a lenient statistical threshold $(p<.01$, uncorrected, $k>30)$ and overlaid on a standardized anatomical template. 


\section{A lateral occipito-temporal ROI}
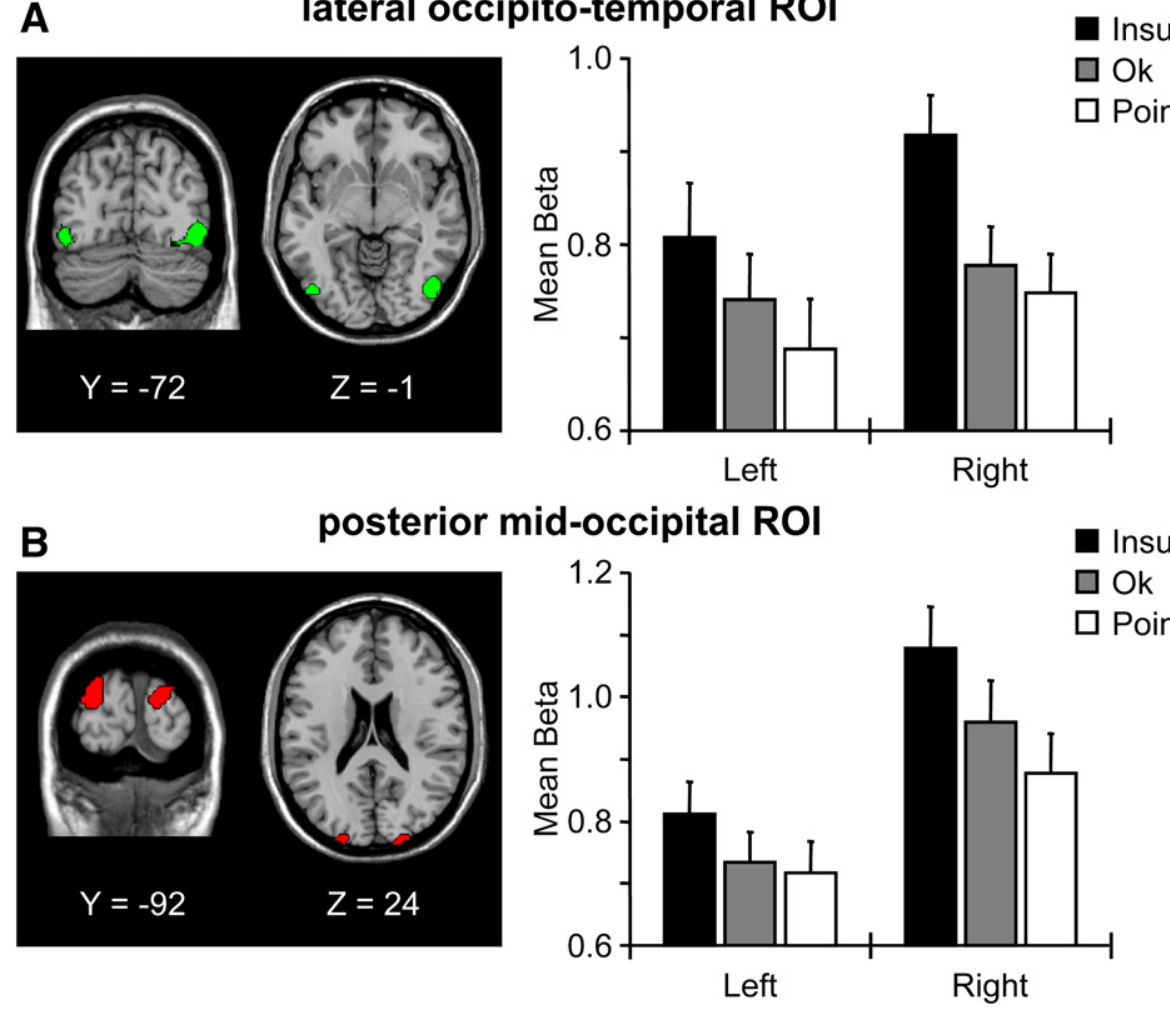

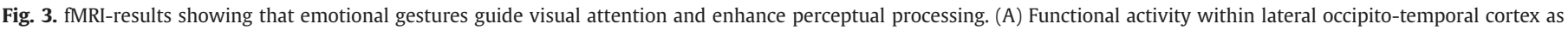

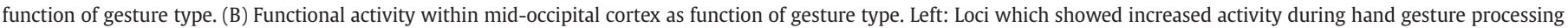

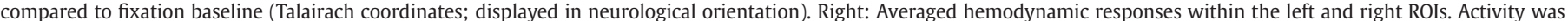

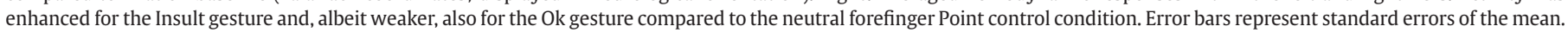

The main effects of Gesture and Laterality were qualified by a significant interaction of both factors $(F(2,30)=12.0, p<.001, \varepsilon=0.93)$ indicating more pronounced emotion modulation over right hemi

A

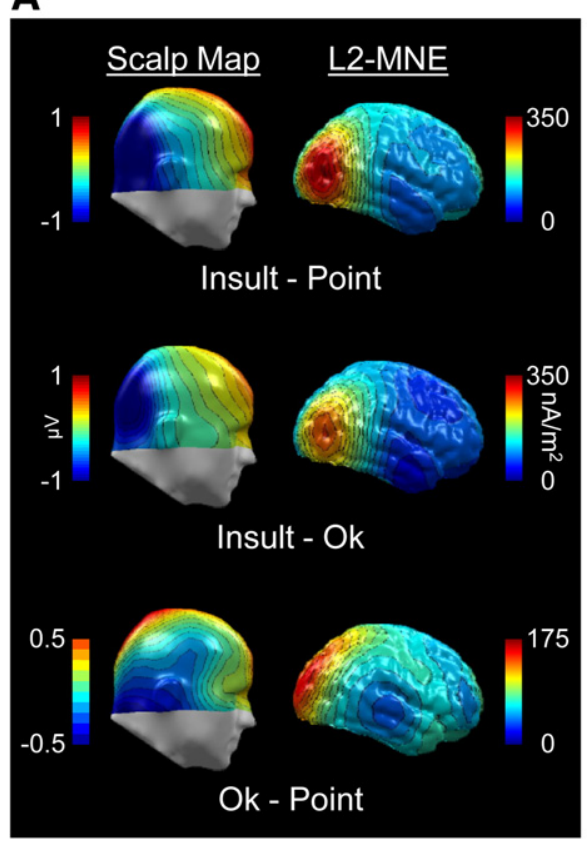

spheric posterior regions (see Fig. 4C). The separate analysis of the left side revealed a significant main effect of Gesture $(F(2,30)=9.7, p<.01$, $\varepsilon=0.88$ ). The Insult gesture elicited an increased posterior negativity

B
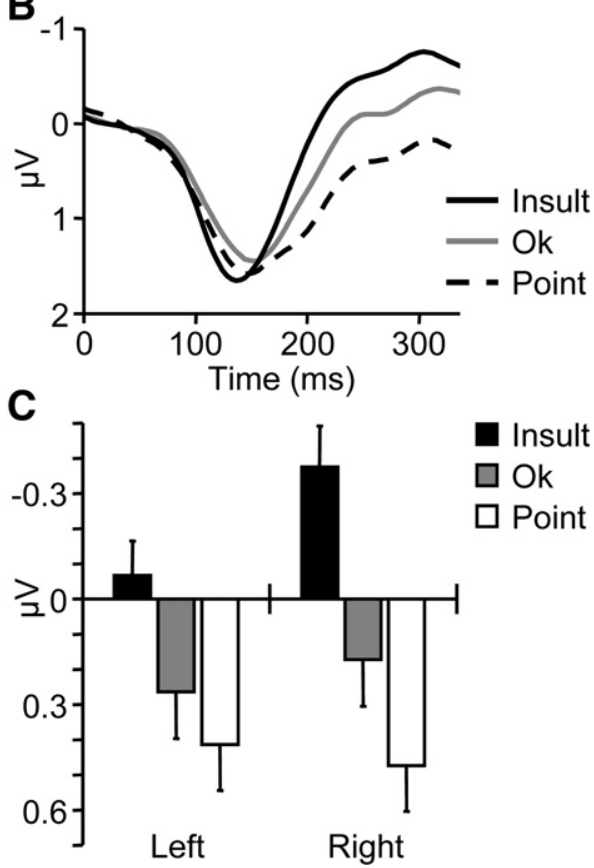

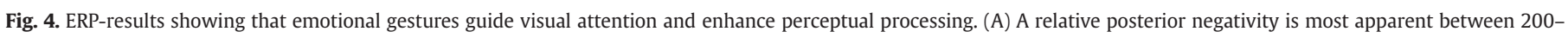

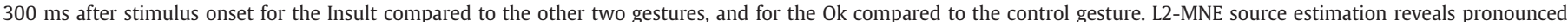

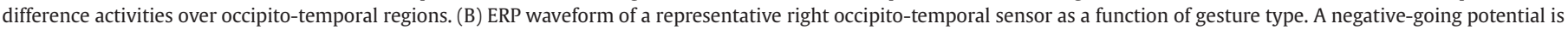

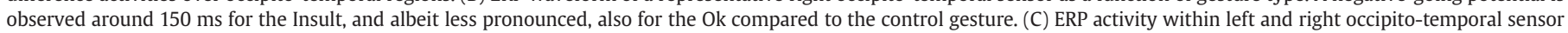
clusters as a function of gesture type. Error bars represent standard errors of the mean. 
relative to the Point $(t(15)=3.9, p<.01)$, and Ok gesture $(t(15)=2.7$, $p<.05)$, which did not differ from each other $(t(15)=1.7$, ns). Analysis of the right side revealed a highly significant main effect of Gesture ( $F$ $(2,30)=29.3, p<.001, \varepsilon=0.76)$. Similar to the left hemisphere, the Insult gesture elicited increased activity relative to the Point $(t(15)=$ $6.2, p<.001)$, and Ok gesture $(t(15)=5.0, p<.001)$. However, on the right side, the Ok gesture elicited a significantly increased EPN compared to the Point gesture $(t(15)=3.6, p<.01)$.

Additionally, L2 Minimum Norm solutions were calculated to provide an estimate of the generator sources of the emotion effects. Selective processing of the Insult gesture was modeled by sources over occipito temporo parietal cortical regions (see Fig. 4A). Albeit less pronounced, similar posterior regions reflected effects of the $\mathrm{Ok}$ compared to the Point gesture.

\section{Gender effects}

The early discrimination of emotional gestures indicated by the EPN was shown by men and women, and no higher order interaction involving Gender was obtained.

\section{Habituation effects}

Considering the factor Time in an extended ANOVA design revealed no significant higher order interaction involving Time. Thus, consis tent with the notion of automaticity, emotional modulation of gesture processing did not habituate across the first and second half of the experiment.

\section{Discussion}

The present studies investigated whether emotional hand gestures guide visual attention and receive preferential processing. In Study 1, functional brain imaging revealed that viewing emotional as com pared to neutral meaningful gestures elicited increased brain activa tion in selected extrastriate visual cortical regions (i.e., posterior mid occipital and lateral occipito temporal). In Study 2, electrophysiolo gical recordings demonstrated the early discrimination of emotional compared to neutral control gestures, appearing already around $150 \mathrm{~ms}$ after stimulus onset. With regard to neural structure and temporal dynamics, the two studies provide converging evidence that emotional hand gestures capture visual attention.

The findings of Study 1 revealed the enhanced perception of emotional hand gestures in posterior mid occipital and lateral occipito temporal regions, which differ with respect to hierarchy and functional specialization of visual object processing (Grill Spector and Malach, 2004). While the posterior mid occipital region repre sents early and mid level visual processing mandatory to object recognition, the lateral occipito temporal cortex belongs to higher level processing areas showing increasing object selectivity. Previous studies determined that a subregion of the lateral occipito temporal cortex, the extrastriate body area (EBA), responds preferentially to pictures of human body parts including arms, hands, and fingers (Downing et al., 2001). Interestingly, the lateral occipito temporal region identified in the present study included activations at/near the EBA coordinates. The emotional enhancement in brain regions devoted to the processing of hand gestures is conceptually similar to observations regarding emotional faces. Specifically, enhancement by emotion was observed in the face sensitive fusiform area comparing fearful to neutral faces (Lewis et al., 2003; Pessoa et al., 2002; Vuilleumier et al., 2001). Moreover, additional areas of emotionally enhanced activation were apparent most pronouncedly in right parietal and somatosensory cortex. This conforms well with the proposed function of right somatosensory cortex in social cognition (Adolphs, 2002), i.e., enabling to recognize emotion from social cues and to form a representation of another person's emotional state. Thus, the notion of predominantly right hemispheric processing of socially relevant signals extends to symbolic emotional hand gestures.
Previous functional imaging studies of gesture processing focused on differences in neural representations of various types of gestures, i.e., contrasting meaningful vs. meaningless, or expressive vs. instru mental gesture representation (Gallagher and Frith, 2004; Grèzes et al., 1999). While another study incorporated emotional gestures, the stimulus materials contained also emotional facial expressions (Lotze et al., 2006). Accordingly, to our knowledge, this is the first investigation specifically examining the processing of symbolic gestures varying in emotional significance. Accepting enhanced activation in brain regions devoted to specific stimulus processing as cardinal sign of selective attention, the present findings strongly suggest that emotional gestures capture visual attention.

A possible difference in processing emotional symbolic gestures and biologically prepared emotion signals is the amount of processing needed to differentiate emotional from neutral cues. In this regard, results from Study 2 showed that the processing of emotional gestures is associated with an early posterior negativity developing around $150 \mathrm{~ms}$ poststimulus and most apparent between $200300 \mathrm{~ms}$. Thus, emotional gestures provoke an early ERP component with respect to latency and topography as has been consistently reported in studies presenting emotional facial expressions and pictures of erotica, threat and attack (Sato et al., 2001; Schupp et al., 2004, 2007). Moreover, a previous fMRI study suggests that emotional enhancement in associative sensory cortices significantly correlates with later brain activity measured in a time window from $400900 \mathrm{~ms}$ (Sabatinelli et al., 2007). Measuring event related brain potentials allows to conclude that symbolic gestures varying in hedonic valence are preferentially processed already during earlier processing stages (i.e., $150300 \mathrm{~ms}$ ) similar to other nonverbal emotional signals. Interestingly, similar findings emerged in studies of emotional language. Across several studies, the reading of pleasant and unpleasant words elicited enhanced EPN amplitudes compared to neutral items (Herbert et al., 2008; Kissler et al., 2007, 2009). Similar to the present results, these effects occurred spontaneously, i.e., in the absence of an explicit task instruction and were stable across multiple stimulus repetitions. Collectively, these findings demonstrate the power of learned emotional significance in modulating the attention capture of symbolic signals during initial stimulus perception.

Study 2 also revealed a pronounced hemispheric asymmetry in that selective emotion processing was most apparent over right occipito temporal regions. These results concur with the fMRI findings of Study 1 and MEG measurements revealing a right hemispheric processing advantage in gesture processing, contrasting meaningful symbolic to meaningless gestures (Nakamura et al., 2004). In contrast, a left hemispheric processing advantage was observed comparing the processing of emotional and neutral words (Kissler et al., 2007). Thus, while gestures and words are symbols depending on shared meaning and cultural convention, left and right lateralized preferential emotion processing suggests hemispheric differences in neural representation.

Both studies suggest a notable asymmetry in the processing of positive and negative hand gestures. The preferential processing of the emotional gestures was most apparent for the negative Insult gesture, significantly enhanced compared to the positive OK gesture in both studies. Such a result is possibly secondary to differences in habituation to positive and negative hand gestures, i.e., more sustained responding to negative compared to positive stimuli (Taylor, 1991). Inconsistent with this hypothesis, enhanced processing of the Insult compared to the Ok gesture was similarly observed in both studies for the second as for the first half of the studies (Schupp et al., 2006). One may further suspect that the asymmetry in emotional gesture processing is specific for women. Previous work exploring gender differences to evolutionary prepared stimuli observed that men showed a bias to respond to positive materials, in particular to stimuli with sexual connotation, while women more strongly reacted to unpleasant materials related to threat and attack (Bradley et al., 
2001; Sabatinelli et al., 2004). Contrary to this hypothesis, no gender differences in the processing of emotional hand gestures were observed here.

To account for the differences of the Insult and Ok gesture, the present data may represent a specific instance of the general phenomenon that bad is stronger than good (Baumeister et al., 2001). The emotional gestures examined here differ with respect to the immediate consequences implied by the gestures (Taylor, 1991). The Insult gesture usually signals the need for immediate responses to prevent harm and injury. In contrast, the Ok gesture often occurs after action is completed. Differences in action disposition may also relate to differences in perceived arousal of the gestures, which were increased for the Insult gesture. This reasoning echoes earlier accounts regarding the processing of angry and happy faces (Schupp et al., 2004), which revealed a similar asymmetry in ERP responses and perceived arousal. Understanding emotion as action sets (Lang et al., 1997), negative cues such as angry expressions and insult gestures, demanding immediate action, may be particularly potent in guiding visual attention.

Overall, the present findings provide converging evidence for the notion that emotional gestures efficiently recruit attentional resources. Enhanced neural activity in visual associative brain regions for emotional gestures may foster the extraction of socially and affectively salient information. The findings implicate ontoge netic experience as a powerful mechanism to guide attentional resources to nonverbal emotion signals. An evolutionary prepared ness to specific emotional gestures, similar to emotional facial expression or body posture (de Gelder, 2006; Öhman and Mineka, 2001), seems unlikely considering that only humans use symbolic gestures. The efficient capture of visual attention by emotional gestures possibly builds upon a more general form of preparedness. Suggested as species specific foundation of human culture, one likely candidate is shared intentionality, i.e., the motivation of humans 'to share emotions, experience, and activities with other persons' (Tomasello et al., 2005). Socially and affectively salient gestures may potentiate the propensity of gestures to direct attentional states to the various conventionalized form of gestures used in a particular culture. Unique features of emotional gestures, such as cultural learning, intentional use, and symbolic nature set them apart from other types of nonverbal emotional communication. Systematic research with emotional gestures enables affective neuroscience to address the interaction of nature and nurture in shaping emotion processing from a broader perspective.

\section{Acknowledgments}

We thank Frank Häcker for his assistance in data collection and analysis and the students of the experimental laboratory course at the University of Konstanz for their contribution in generating the stimulus materials. This research was supported by Grants Schu1074/7 4 and Schu 1074/11 1 from the German Research Society to H. T. Schupp.

\section{References}

Adolphs, R., 2002. Neural systems for recognizing emotion. Curr. Opin. Neurobiol. 12 169-177.

Archer, D., 1997. Unspoken diversity: cultural differences in gestures. Qual. Sociol. 20, 79-105.

Baumeister, R.F., Bratslavsky, E., Finkenauer, C., Vohs, K.D., 2001. Bad is stronger than good. Rev. Gen. Psychol. 5, 323-370.

Bradley, M.M., Lang, P.J., 1994. Measuring emotion: the self-assessment manikin and the semantic differential. J. Behav. Ther. Exp. Psychiatry 25, 49-59.

Bradley, M.M., Codispoti, M., Sabatinelli, D., Lang, P.J., 2001. Emotion and motivation II: Sex differences in picture processing. Emotion 1, 300-319.

Bradley, M.M., Sabatinelli, D., Lang, P.J., Fitzsimmons, J.R., King, W., Desai, P., 2003. Activation of the visual cortex in motivated attention. Behav. Neurosci. 117, 369-380.

Buck, R., VanLear, C.A., 2002. Verbal and nonverbal communication: distinguishing symbolic, spontaneous, and pseudo-spontaneous nonverbal behavior. J. Commun. 52, 522-541.
Codispoti, M., Ferrari, V., Junghöfer, M., Schupp, H.T., 2006. The categorization of natural scenes: brain attention networks revealed by dense sensor ERPs. NeuroImage 32, 583-591.

de Gelder, B., 2006. Towards the neurobiology of emotional body language. Nat. Rev., Neurosci. 7, 242-249.

de Gelder, B., Snyder, J., Greve, D., Gerard, G., Hadjikhani, N., 2004. Fear fosters flight: a mechanism for fear contagion when perceiving emotion expressed by a whole body. Proc. Natl. Acad. Sci. U. S. A. 101, 16701-16706.

Delorme, A., Rousselet, G.A., Mace, M.J., Fabre-Thorpe, M., 2004. Interaction of top-down and bottom-up processing in the fast visual analysis of natural scenes. Cogn. Brain Res. 19, 103-113.

Downing, P.E., Jiang, Y., Shuman, M., Kanwisher, N., 2001. A cortical area selective for visual processing of the human body. Science 293, 2470-2473.

Flaisch, T., Junghöfer, M., Bradley, M.M., Schupp, H.T., Lang, P.J., 2008. Rapid picture processing: affective primes and targets. Psychophysiology 45, 1-10.

Friston, K.J., Holmes, A.P., Worsley, K.J., Poline, J.B., Frith, C., Frackowiak, R.S.J., 1995. Statistical parametric maps in functional imaging: a general linear approach. Hum. Brain Mapp. 2, 189-210.

Gallagher, H.L., Frith, C.D., 2004. Dissociable neural pathways for the perception and recognition of expressive and instrumental gestures. Neuropsychologia 42, $1725-1736$.

Grèzes, J., Costes, N., Decety, J., 1999. The effects of learning and intention on the neural networks involved in the perception of meaningless actions. Brain 122, 1875-1887.

Grill-Spector, K., Malach, R., 2004. The human visual cortex. Annu. Rev. Neurosci. 27, 649-677.

Hadjikhani, N., de Gelder, B., 2003. Seeing fearful body expressions activates the fusiform cortex and amygdala. Curr. Biol. 13, 2201-2205.

Hauk, O., 2004. Keep it simple: a case for using classical minimum norm estimation in the analysis of EEG and MEG data. NeuroImage 21, 1612-1621.

Herbert, C., Junghöfer, M., Kissler, J., 2008. Event related potentials to emotional adjectives during reading. Psychophysiology 45, 487-498.

Junghöfer, M., Peyk, P., 2004. Analysis of electrical potentials and magnetic fields of the brain. Matlab Select 2, 24-28. EMEGS software is freely available at $h t t p: / / w w w$. emegs.org.

Junghöfer, M., Elbert, T., Tucker, D.M., Rockstroh, B., 2000. Statistical control of artifacts in dense array EEG/MEG studies. Psychophysiology 37, 523-532.

Junghöfer, M., Bradley, M.M., Elbert, T.R., Lang, P.J., 2001. Fleeting images: a new look at early emotion discrimination. Psychophysiology 38, 175-178.

Junghöfer, M., Schupp, H.T., Stark, R., Vaitl, D., 2005. Neuroimaging of emotion: empirical effects of proportional global signal scaling in fMRI data analysis. NeuroImage 25, 520-526.

Junghöfer, M., Sabatinelli, D., Bradley, M.M., Schupp, H.T., Elbert, T.R., Lang, P.., 2006. Fleeting images: rapid affect discrimination in the visual cortex. NeuroReport 17, 225-229.

Kissler, J., Herbert, C., Peyk, P., Junghöfer, M., 2007. Buzzwords: early cortical responses to emotional words during reading. Psychol. Sci. 18, 475-480.

Kissler, J., Herbert, C., Winkler, I., Junghöfer, M., 2009. Emotion and attention in visual word processing: an ERP study. Biol. Psychol. 80, 75-83.

Lancaster, J.L., Woldorff, M.G., Parsons, L.M., Liotti, M., Freitas, C.S., Rainey, L., Kochunov, P.V., Nickerson, D., Mikiten, S.A., Fox, P.T., 2000. Automated Talairach atlas labels for functional brain mapping. Hum. Brain Mapp. 10, 120-131.

Lang, P.J., Bradley, M.M., Cuthbert, B.N., 1997. Motivated attention: affect, activation, and action. In: Lang, P.J., Simons, R.F., Balaban, M. (Eds.), Attention and Emotion: Sensory and Motivational Processes. Erlbaum, Mahwah, NJ, pp. 97-135.

Leppänen, J.M., Kauppinen, P.K., Peltola, M.J., Hietanen, J.K., 2007. Differential electrocortical responses to increasing intensities of fearful and happy emotional expressions. Brain Res. 1166, 103-109.

Lewis, S., Thoma, R.J., Lanoue, M.D., Miller, G.A., Heller, W., Edgar, C., Huang, M., Weisend, M.P., Irwin, J., Paulson, K., Canive, J.M., 2003. Visual processing of facial affect. NeuroReport 14, 1841-1845.

Lotze, M., Heymans, U., Birbaumer, N., Veit, R., Erb, M., Flor, H., Halsband, U., 2006. Differential cerebral activation during observation of expressive gestures and motor acts. Neuropsychologia 44, 1787-1795.

Morris, D., 1994. Bodytalk: The Meaning of Human Gestures. Crown Publishers, New York.

Morris, D., Collett, P., Marsh, P., O'Shaughnessy, M., 1979. Gestures: Their Origins and Distribution. Jonathan Cape, London.

Nakamura, A., Maess, B., Knosche, T.R., Gunter, T.C., Bach, P., Friederici, A.D., 2004. Cooperation of different neuronal systems during hand sign recognition. NeuroImage 23, 25-34.

Öhman, A., Mineka, S., 2001. Fears, phobias, and preparedness: toward an evolved module of fear and fear learning. Psychol. Rev. 108, 483-522.

Öhman, A., Flykt, A., Lundqvist, D., 2000. Unconscious emotion: evolutionary perspectives, psychophysiological data and neuropsychological mechanisms. In: Lane, R.D., Nadel, L. (Eds.), Cognitive Neuroscience of Emotion. Oxford University Press, New York, pp. 296-327.

Peelen, M.V., Atkinson, A.P., Andersson, F., Vuilleumier, P., 2007. Emotional modulation of body-selective visual areas. Soc. Cogn. Affect. Neurosci. 2, 274-283.

Pessoa, L., McKenna, M., Gutierrez, E., Ungerleider, L.G., 2002. Neural processing of emotional faces requires attention. Proc. Natl. Acad. Sci. U. S. A. 99, $11458-11463$.

Pika, S., Liebal, K., Call, J., Tomasello, M., 2005. The gestural communication of apes. Gesture 5, 41-56.

Potts, G.F., Tucker, D.M., 2001. Frontal evaluation and posterior representation in target detection. Cogn. Brain Res. 11, 147-156. 
Pruessmann, K.P., Weiger, M., Scheidegger, M.B., Boesiger, P., 1999. SENSE: sensitivity encoding for fast MRI. Magn. Reson. Med. 42, 952-962.

Rorden, C., Brett, M., 2000. Stereotaxic display of brain lesions. Behav. Neurol. 12 191-200.

Sabatinelli, D., Bradley, M.M., Fitzsimmons, J.R., Lang, P.J., 2005. Parallel amygdala and inferotemporal activation reflect emotional intensity and fear relevance. NeuroImage 24, 1265-1270.

Sabatinelli, D., Flaisch, T., Bradley, M.M., Fitzsimmons, J.R., Lang, P.J., 2004. Affective picture perception: gender differences in visual cortex? NeuroReport 15, 1109-1112.

Sabatinelli, D., Lang, P.J., Keil, A., Bradley, M.M., 2007. Emotional perception: correlation of functional MRI and event-related potentials. Cereb. Cortex 17, 1085-1091.

Sato, W., Kochiyama, T., Yoshikawa, S., Matsumura, M., 2001. Emotional expression boosts early visual processing of the face: ERP recording and its decomposition by independent component analysis. NeuroReport 12, 709-714.

Schupp, H.T., Junghöfer, M., Weike, A.I., Hamm, A.O., 2003. Emotional facilitation of sensory processing in the visual cortex. Psychol. Sci. 14, 7-13.
Schupp, H.T., Öhman, A., Junghöfer, M., Weike, A.I., Stockburger, J., Hamm, A.O., 2004. The facilitated processing of threatening faces: an ERP analysis. Emotion 4 189-200.

Schupp, H.T., Stockburger, J., Codispoti, M., Junghöfer, M., Weike, A.I., Hamm, A.O., 2006. Stimulus novelty and emotion perception: the near absence of habituation in the visual cortex. NeuroReport 17, 365-369.

Schupp, H.T., Stockburger, J., Codispoti, M., Junghöfer, M., Weike, A.I., Hamm, A.O., 2007 Selective visual attention to emotion. J. Neurosci. 27, 1082-1089.

Taylor, S.E., 1991. Asymmetrical effects of positive and negative events: the mobilization-minimization hypothesis. Psychol. Bull. 110, 67-85.

Tomasello, M., Carpenter, M., Call, J., Behne, T., Moll, H., 2005. Understanding and sharing intentions: the origins of cultural cognition. Behav. Brain Sci. 28, 675-691 discussion 691-735.

Vuilleumier, P., 2005. How brains beware: neural mechanisms of emotional attention. Trends Cogn. Sci. 9, 585-594.

Vuilleumier, P., Armony, J.L., Driver, J., Dolan, R.J., 2001. Effects of attention and emotion on face processing in the human brain: an event-related fMRI study. Neuron 30 , 829-841. 